Check for updates

Cite this: RSC Adv., 2019, 9, 14868

Received 21st February 2019

Accepted 27th April 2019

DOI: $10.1039 / c 9 r a 01309 a$

rsc.li/rsc-advances

\section{Improvement of perovskite crystallinity by omnidirectional heat transfer via radiative thermal annealing ${ }^{\dagger}$}

\author{
Jiyoon Park, $t^{\mathrm{a}}$ Jin Woo Choi, (D) $\dot{t}^{\mathrm{b}}$ Woochul Kim, ${ }^{a}$ Ryeri Lee, ${ }^{\mathrm{a}}$ Hee Chul Woo, (D) ${ }^{\mathrm{b}}$ \\ Jisoo Shin, ${ }^{b}$ Hyeonghun Kim, ${ }^{a}$ Yeong Jun Son, ${ }^{a}$ Ji Young Jo, (DD ${ }^{a}$ Heon Lee, ${ }^{c}$ \\ Sooncheol Kwon, ${ }^{d}$ Chang-Lyoul Lee (D) *b and Gun Young Jung (D) *a
}

\begin{abstract}
As promising photo-absorbing materials for photovoltaics, organic-inorganic hybrid perovskite materials such as methylammonium lead iodide and formamidinium lead iodide, have attracted lots of attention from many researchers. Among the various factors to be considered for high power conversion efficiency (PCE) in perovskite solar cells (PSCs), increasing the grain size of perovskite is most important. However, it is difficult to obtain a highly crystalline perovskite film with large grain size by using the conventional hot-plate annealing method because heat is transferred unidirectionally from the bottom to the top. In this work, we presented radiative thermal annealing (RTA) to improve the structural and electrical properties of perovskite films. Owing to the omnidirectional heat transfer, swift and uniform nuclei formation was possible within the perovskite film. An average grain size of $500 \mathrm{~nm}$ was obtained, which is 5 times larger than that of the perovskite film annealed on a hot-plate. This perovskite film led to an enhancement of photovoltaic performance of PSCs. Both short-circuit current density and PCE of the PSCs prepared by RTA were improved by $10 \%$, compared to those of PSCs prepared by hot-plate annealing.
\end{abstract}

\section{Introduction}

Solar energy is considered an eco-friendly next-generation renewable energy. Among the various types of solar cells, perovskite solar cells (PSCs) have attracted considerable attention over the past decade due to their outstanding optical and electrical properties, such as high light absorption coefficient in the visible range, low exciton binding energy under $50 \mathrm{meV}$, high ambipolar charge mobility as well as solution processability. ${ }^{1-9}$ The power conversion efficiency (PCE) of PSCs has

${ }^{a}$ School of Materials Science and Engineering (SMSE), Gwangju Institute of Science and Technology (GIST), 123 Cheomdangwagi-ro, Buk-gu, Gwangju 61005, Republic of Korea.E-mail: gyjung@gist.ac.kr

${ }^{b}$ Advanced Photonics Research Institute (APRI), Gwangju Institute of Science and Technology (GIST), 123 Cheomdangwagi-ro, Buk-gu, Gwangju 61005, Republic of Korea. E-mail:vsepr@gist.ac.kr

${ }^{c}$ Department of Materials Science and Engineering, Korea University, Seoul 02841, Republic of Korea

${ }^{d}$ Research Institute of Solar and Sustainable Energies (RISE), Gwangju Institute of Science and Technology (GIST), 123 Cheomdangwagi-ro, Buk-gu, Gwangju 61005, Republic of Korea

$\dagger$ Electronic supplementary information (ESI) available: The structural (SEM, XRD) characterization of RTA-perovskite films, photovoltaic performance of RTA-PSCs at different annealing times, temperature-dependence of PL spectra of perovskite films prepared by hot-plate annealing and RTA, and schematic of PSC fabrication via RTA. See DOI: 10.1039/c9ra01309a

$\ddagger$ These authors equally contributed to this work. been dramatically enhanced, reaching $\sim 22 \%$. Furthermore, the PCE of PSCs is expected to surpass that of silicon solar cells within 10 years..$^{10-17}$

Several factors, such as halide composition control for bandgap tuning of the perovskite photo-active layer, ${ }^{18}$ high crystallinity of the perovskite film with large grain size ${ }^{19}$ and optimization of energy band alignment in the device structure, ${ }^{20}$ must be improved to the satisfactory level to realize high PCE in PSCs. Among these factors, the crystallinity and grain size of perovskite, which are strongly related to exciton dissociation, ${ }^{21}$ charge transport ${ }^{22-24}$ and extraction efficiency, ${ }^{25,26}$ are extremely important to enhance the photovoltaic performance of PSCs. High crystallinity in a perovskite film signifies a low density of defects with less grain boundaries (GBs). It suppresses the energy band distortion induced by GBs of the perovskite film, ${ }^{27}$ resulting in efficient exciton dissociation by minimizing carrier recombination at the GBs. Perovskite films with larger grains have less GBs, which improve their charge transport and extraction efficiency ${ }^{26}$ by suppressing charge recombination and trapping. ${ }^{19}$

To realize a highly crystalline perovskite film with large grain size, various methods have been reported, including one-step spin-coating with anti-solvent dripping, ${ }^{28,29}$ deposition by an evaporation method under vacuum conditions ${ }^{13,30}$ and blade coating. ${ }^{31}$ In addition, control of the crystallinity and grain size of perovskite film through various post-annealing methods has 
been investigated..$^{32,33}$ In general, as-deposited perovskite films are subsequently annealed on a hot-plate to form the crystalline perovskite by evaporating the solvent. ${ }^{10-17}$ However, this method has a critical limitation of unidirectional heat transfer from the bottom; the annealing temperature is inhomogeneous between the top and bottom sides of the perovskite film. Due to the lack of uniform heat distribution within the perovskite film, the hotplate annealing method yields perovskite films with inferior crystallinity and smaller grain size compared to the other reported annealing methods and suffers from poor reproducibility. Therefore, there is a great need to develop new postannealing methods to replace the hot-plate annealing method for obtaining highly crystalline perovskite films with large grains.

Recently, two research groups reported new post-annealing methods using a light as an energy source and non-direct thermal heating; Jeon et al. introduced a near-infrared (NIR) laser annealing method and obtained a large grain size $(\sim 300$ $\mathrm{nm}$ ) in perovskite film. ${ }^{32} \mathrm{~F}$. Huang et al. reported a dual-flash annealing process, in which the perovskite film was treated via a 2-step annealing process involving a low-pressure flash evaporation stage followed by a high-temperature flash annealing step. ${ }^{33}$ The PSCs based on these films exhibited enhanced photovoltaic (PV) performances ( 19\%). Although the abovementioned methods are significant for improving the crystallinity along with large grain size, there are limitations, such as long processing time and expensive equipment.

In addition, many studies have also been reported on annealing for as-deposited perovskite films by using rapid thermal annealing techniques. In general, the rapid thermal annealing methods have been widely used to improve the structural and electrical properties of semiconducting materials. ${ }^{34,35}$ These techniques can raise the temperature rapidly by using various heating sources such as intense pulsed light, ${ }^{32,36}$ flash light, ${ }^{33}$ infrared (IR) radiation, ${ }^{37,38}$ etc. Among them, the radiative annealing using the IR lamp is advantageous for transferring the heat omnidirectionally, allowing uniform heat distribution within the thin films but, it was barely used in PSCs. V. L. Pool et al. utilized the radiative thermal annealing (hereafter abbreviated as RTA) technique in perovskite film annealing stage to explain the decomposition kinetics of $\mathrm{FAPbI}_{3}$ depending on the RTA temperature and heating time. ${ }^{37}$ B. Dou et al. also used the RTA method for annealing as-deposited perovskite films to see the effect of processing conditions, i.e. anti-solvent, humidity, and annealing conditions, on the film quality of $\mathrm{MAPbI}_{3}$ through in situ XRD analysis. ${ }^{38}$

In this study, we applied a radiative thermal annealing (RTA) to improve the crystallinity and grain size of perovskite films. Differences in crystallinity and grain size of the perovskite films prepared by these two different post-annealing methods (RTA $v s$. hot plate annealing) and their effects on the device performance of PSCs were investigated. 20 PSCs were fabricated by each method and their PV performances were statistically characterized. In addition, the interfacial and optical (exciton) characteristics of perovskite films, such as charge transport, exciton binding energy and charge dissociation, were also investigated to verify the origin for the enhanced PV performance by the RTA.

\section{Experimental section}

\subsection{Fabrication of perovskite solar cells using the RTA- perovskite}

A fluorine-doped tin oxide (FTO) substrate was cleaned for $15 \mathrm{~min}$ in sequence of acetone, isopropyl alcohol, deionized water and dried using a nitrogen gun. The substrate was further cleaned by UV-ozone for $15 \mathrm{~min}$. After UV-ozone treatment, a compact $\mathrm{TiO}_{2}$ layer acting as a hole blocker was deposited onto the cleaned FTO substrate by spin-coating a $\mathrm{TiO}_{2}$ stock solution of $0.15 \mathrm{M}$ titanium butoxide in 1-butanol at $2000 \mathrm{rpm}$ for $40 \mathrm{~s}$. After spin-coating, the substrate was dried on a hot-plate at $125{ }^{\circ} \mathrm{C}$ for $5 \mathrm{~min}$ to remove the residual solvent. Then, the substrate was annealed at $550{ }^{\circ} \mathrm{C}$ for $30 \mathrm{~min}$ in a furnace to transform the $\mathrm{TiO}_{2}$ phase from rutile to anatase. The mesoporous $\mathrm{TiO}_{2}$ layer as an electron-transport layer (ETL) was formed by spin-coating a $\mathrm{TiO}_{2}$ paste, which was composed of $30 \mathrm{~nm}$ nanoparticles (NPs) in 2-methoxyethanol (1:5 weight ratio), at $4000 \mathrm{rpm}$ for $15 \mathrm{~s}$ onto the $\mathrm{TiO}_{2}$ blocking layer. The sample was dried at $125{ }^{\circ} \mathrm{C}$ for $5 \mathrm{~min}$ and then annealed at $550{ }^{\circ} \mathrm{C}$ for $1 \mathrm{~h}$ to burn out the polymer ligands wrapping around the $\mathrm{TiO}_{2} \mathrm{NPs}$.

The perovskite precursor solution was prepared by mixing 1.2 $\mathrm{M} \mathrm{PbI}_{2}$ (99\%, TCI), $1.02 \mathrm{M}$ methylammonium iodide (MAI, 98\%, Sigma Aldrich), $0.18 \mathrm{M}$ formamidinium iodide (FAI, 99\%, TCI) and 1.2 M DMSO (99\%, Sigma Aldrich) in DMF (99\%, Sigma Aldrich). The solution was then stirred at room temperature for $6 \mathrm{~h}$. An approximately $300 \mathrm{~nm}$-thick $\left(\mathrm{MAPbI}_{3}\right)_{0.85}\left(\mathrm{FAPbI}_{3}\right)_{0.15}$ layer was deposited by spin-coating the precursor solution at $3500 \mathrm{rpm}$ for $40 \mathrm{~s}$. Just before spin-coating was finished, $80 \mu \mathrm{L}$ of toluene was dropped to instantaneously remove the DMF within the perovskite film. The as-deposited perovskite films were annealed in a radiative thermal annealing (RTA) system under $\mathrm{N}_{2}$ atmosphere. Fig. S1a (ESI $\dagger$ ) shows the schematic presentation of the RTA steps. Chamber was heated up to $120{ }^{\circ} \mathrm{C}$ for $30 \mathrm{~s}$ with a ramping rate of $4{ }^{\circ} \mathrm{C} \mathrm{s}^{-1}$. After reaching the set temperature, the film was annealed at $120{ }^{\circ} \mathrm{C}$ for $10 \mathrm{~min}$. A spiro-OMeTAD layer as a hole-transport layer (HTL) was deposited onto the perovskite layer by spincoating at $2000 \mathrm{rpm}$ for $20 \mathrm{~s}$. The spiro-OMeTAD solution was prepared by dissolving $72.3 \mathrm{mg}$ of spiro-OMeTAD in $1 \mathrm{~mL}$ of chlorobenzene and then adding $28.8 \mu \mathrm{L}$ of 4-tert-butylpyridine and $17.5 \mu \mathrm{L}$ of a stock solution of lithium bis(trifluoromethylsulfonyl) imide (Li-TFSI $520 \mathrm{mg}$ in $1 \mathrm{~mL}$ of acetonitrile). After spin-coating the HTL, the samples were stored in a desiccator for $24 \mathrm{~h}$ to oxidize the spiro-OMeTAD for improving the hole extraction and transport properties. ${ }^{39}$ Finally, an 80 nm-thick gold electrode was deposited by thermal evaporation. All processes for PSCs fabrication were performed in a glove box filled with high purity $\mathrm{N}_{2}$ gas except when transporting the sample to RTA apparatus. All procedures are illustrated in Fig. S1b (ESI†). A schematic and a cross-sectional scanning electron microscopy image of a perovskite solar cell are shown in Fig. S1c and S1d (ESI $\dagger$ ).

\subsection{Crystal structure and morphology characterization of perovskite films}

The crystalline phase of perovskite films was characterized by $\mathrm{X}$ ray diffraction (XRD) using a $\mathrm{CuK}_{\alpha}$ radiation source $(40 \mathrm{kV}, 40$ 
mA, Advanced D8, Bruker, Germany). The surface crystalline phase of perovskite film was analyzed by grazing incidence XRD (GIXRD) using above mentioned XRD equipment. In GIXRD measurements, the incidence angle of X-ray was $0.1^{\circ}$. The morphology of the perovskite films was observed with fieldemission scanning electron microscopy (FE-SEM, JEOL 2020F) with an accelerating voltage of $10 \mathrm{keV}$.

\subsection{Measurements}

2.3.1 Photovoltaic performance measurements. The current density versus voltage $(J-V)$ characteristics of PSCs were measured by using a Keithley Model 2400 source meter under an AM 1.5G illumination with a power density of $100 \pm 2.5 \mathrm{~mW}$ $\mathrm{cm}^{-2}$ (SANEI solar simulator, Class A). The external quantum efficiency (EQE) of PSCs was measured by an incident photon-toelectron conversion efficiency (IPCE) measurement (QE-IPCE 3000). The PV performance of PSCs was measured under ambient atmosphere without any passivation. The Nyquist plots were measured by electrochemical impedance spectroscopy (EIS, FRA2 $\mu$ AUTOLAB type III) over a frequency range of $1 \mathrm{~Hz}$ to $1 \mathrm{MHz}$ at the open circuit voltage of each cell under dark condition.

2.3.2 Optical property measurements. The temperaturedependent steady-state photoluminescence (PL) spectra of perovskite films were measured by using a monochromator (SP-2150i, Acton), a photomultiplier tube (PMT, Acton PD471) and pulsed diode-laser head (LDH-P-C-405, PicoQuant) system. The temperature was varied from $60 \mathrm{~K}$ to $200 \mathrm{~K}$. The time-resolved PL (TR-PL) of perovskite films was monitored at $775 \mathrm{~nm}$ by using a TCSPC module (PicoHarp 260, PicoQuant) with a micro channel plate photomultiplier tube (MCP-PMT, R3809U-50, Hamamatsu), a $400 \mathrm{~nm}$ picosecond pulsed laser (LDH-P-C-405, PicoQuant) coupled with laser-diode driver (PDL 800-B, PicoQuant).

\section{Result and discussion}

In general, X-ray diffraction (XRD) patterns are utilized to analyze the crystalline structure and degree of crystallinity of films. Fig. 1 shows the crystallographic characterization of perovskite films prepared by two different post-annealing methods. To precisely compare the effect of each annealing method on the crystallographic property, optimization of two post-annealing processes was first conducted. As several research groups reported that heat treatment at $120{ }^{\circ} \mathrm{C}$ is the optimum condition for post-annealing of perovskite films on a hot-plate (HPA-perovskite), ${ }^{\mathbf{4 0}-43}$ we also fixed the annealing temperature at $120{ }^{\circ} \mathrm{C}$ for the radiative thermal annealing process (RTA-perovskite) so that we can carry out direct comparison. In the post-annealing process using a hot-plate, the annealing temperature and time were fixed at $120{ }^{\circ} \mathrm{C}$ and $10 \mathrm{~min}$, respectively, in accordance with the references. ${ }^{\mathbf{4 0}-43}$ Top-view scanning electron microscopy (SEM) images of perovskite films at various RTA times (from $1 \mathrm{~min}$ to $20 \mathrm{~min}$ ) and their corresponding XRD patterns are shown in Fig. S2a and S2b (ESI $\dagger$ ). There was no significant difference in the SEM images with increasing the RTA time; however, the XRD results showed a considerable difference. After $10 \mathrm{~min}$ of post- annealing, a new diffraction peak emerged at $12.7^{\circ}$, corresponding to the (001) plane of $\mathrm{PbI}_{2} \cdot{ }^{44}$ The intensity of $\mathrm{PbI}_{2}$ peak increased with increasing the RTA time. It was previously reported that a small amount of $\mathrm{PbI}_{2}$ can act as a passivation layer to prevent recombination at the interface between the perovskite and the hole-transport layer (HTL), ${ }^{45,46}$ which could contribute to improve the short-circuit current density $\left(J_{\mathrm{sc}}\right)$ in the PSCs. However, excess $\mathrm{PbI}_{2}$ has adverse effects on the photovoltaic (PV) performances of PSCs when annealed for more than $10 \mathrm{~min}$, which will be explained in more detail in the device performance section. Based on the SEM and XRD results shown in Fig. S2a and S2b (ESI $\dagger$ ), annealing at $120^{\circ} \mathrm{C}$ for $10 \mathrm{~min}$ was chosen as the optimum condition for the RTA-perovskite. The PV parameters and their statistical distribution depending on the RTA time are shown in Table S1 and Fig. S2c (ESI). $\dagger$

Fig. 1a shows XRD patterns of perovskite films annealed by two different methods. The prominent XRD peaks in both films appeared at $14.08^{\circ}$ and $28.38^{\circ}$, corresponding to the (110) and (220) planes of the perovskite, respectively. ${ }^{44}$ Compared to those in HPA-perovskite, the intensity of main (110) peak at $14.08^{\circ}$ in RTA-perovskite was higher and the full-width at half-maximum (FWHM) value was smaller, indicating that the crystallinity of the RTA-perovskite was better than that of the HPA-perovskite. Interestingly, there was no $\mathrm{PbI}_{2}$ diffraction peak at $12.7^{\circ}$ in the HPA-perovskite. We assumed that it resulted from the difference in heat transfer mechanisms between hot plate annealing and RTA system. In hot plate annealing system, the perovskite film received less energy from the heating source since the heat travelled through glass, fluorine-doped tin oxide (FTO), electron transporting layer (ETL) to reach the perovskite film. However, in RTA system, since the heat was transferred directly to the perovskite film, it received all energy from the heating source without any loss. ${ }^{38}$ As a result, we predict that the degree of methylammonium iodide (MAI) decomposition is different between hot plate annealing and RTA system, resulting in the formation of a small amount $\mathrm{PbI}_{2}$ only in RTA. Fig. $1 \mathrm{~b}$ and $\mathrm{c}$ compare top-view SEM images of perovskite films prepared by two different post-annealing methods. The insets are low magnification SEM images of both perovskite films. The (a)

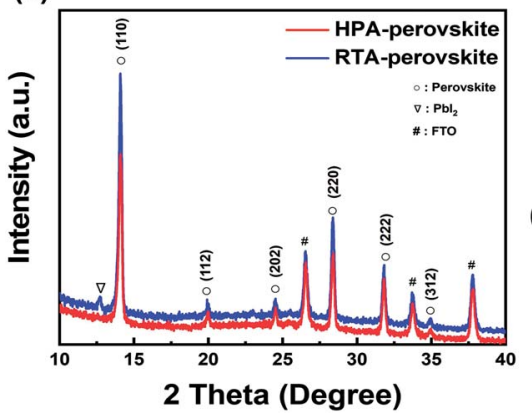

(b)

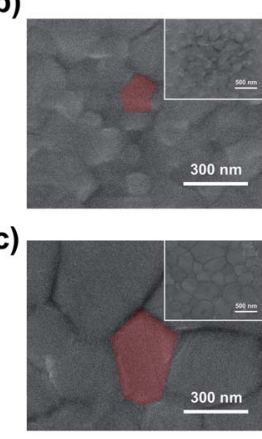

Fig. 1 Structural characterization of perovskite films prepared by two different annealing methods. (a) XRD patterns of two perovskite films prepared by two different post-annealing processes. Top-view FESEM images of (b) HPA-perovskite film and (c) RTA-perovskite film. The red-colored area represents one grain and the inset image is lowmagnified FE-SEM image. 
average grain size of RTA-perovskite was measured as approximately $500 \mathrm{~nm}$, however, sub-100 nm grains were observed in the HPA-perovskite.

The grain size difference between the RTA- and HPA-perovskite was resulted from the difference in nucleation rate by heat transfer mechanism. ${ }^{47}$ In general, the grains are developed through three steps; (i) nucleation, (ii) subsequent crystal growth at the nucleus and (iii) merging of neighboring crystals. Of these steps, the nucleation process occurs when the embryo overcomes its activation energy $(\Delta G)$ for making nuclei. ${ }^{48}$ In the hot plateannealing, the temperature gradient occurred within the asdeposited perovskite film since the thermal energy was delivered from the bottom to upper layer by unidirectional heat transfer via conduction (left scheme in Fig. 2a). Thus, the nuclei were initially formed only at the bottom side of perovskite film. Consequentially, a relatively longer time was required for nucleation in the whole perovskite film. Therefore, it shortened the time for the growth of crystals and their merging into grains during the limited annealing time, resulting in a smaller grain size.

Besides, the movement of precursor ions was largely dependent on their position within the as-deposited perovskite film due to the unidirectional heat transfer from bottom side. Compared to the bottom part of perovskite film, which received huge amount of thermal energy from hot-plate via conduction, the movement of precursor ions at the top side was relatively suppressed due to the less thermal energy. It reduced the chance of the precursor ions sticking to the embryos, rendering less chance for nuclei formation. Therefore, this thermal energy distribution within the film induced uneven nuclei formation and accordingly grain growth with different sizes along the thickness of perovskite film. ${ }^{47}$ These phenomena including slow nucleation and suppressed migration of ions generated perovskite grains of sub-100 nm size by hot-plate annealing (Fig. 1b).

However, in the RTA process, thermal energy distribution within the as-deposited perovskite film was much uniform than that of the hot-plate annealing process owing to the omnidirectional heat transfer via radiation (right scheme in Fig. 2a). Active movement of precursor ions produced uniform nuclei in a relatively short time within the as-deposited perovskite film regardless of the position. Therefore, more thermal energy can be used up for the crystal growth and their merging into a grain in the same annealing time, inducing larger perovskite grains of approximately $500 \mathrm{~nm}$ size (Fig. 1c).

To further check the nucleation and grain growth at the film surface in initial annealing state of RTA process, grazingincidence XRD (GIXRD) measurements were conducted with the HPA- and RTA-treated films with an incidence angle of $0.1^{\circ}$. In general, as the GIXRD uses a small incidence angle for incoming X-ray, the penetration depth of X-ray is limited to the surface and most scattering signals mainly comes from it. ${ }^{49}$ The heat treatment of perovskite films was performed for only $30 \mathrm{~s}$ to check the initial nuclei creation at the surface region. Fig. $2 \mathrm{~b}$ shows the GIXRD patterns of HPA- and RTA-perovskite in initial annealing stage. The intensity of (110) main peak of RTAperovskite was higher than that of HPA-perovskite, demonstrating that crystallization process including nucleation and crystal growth occurred more actively at the surface by the RTA.
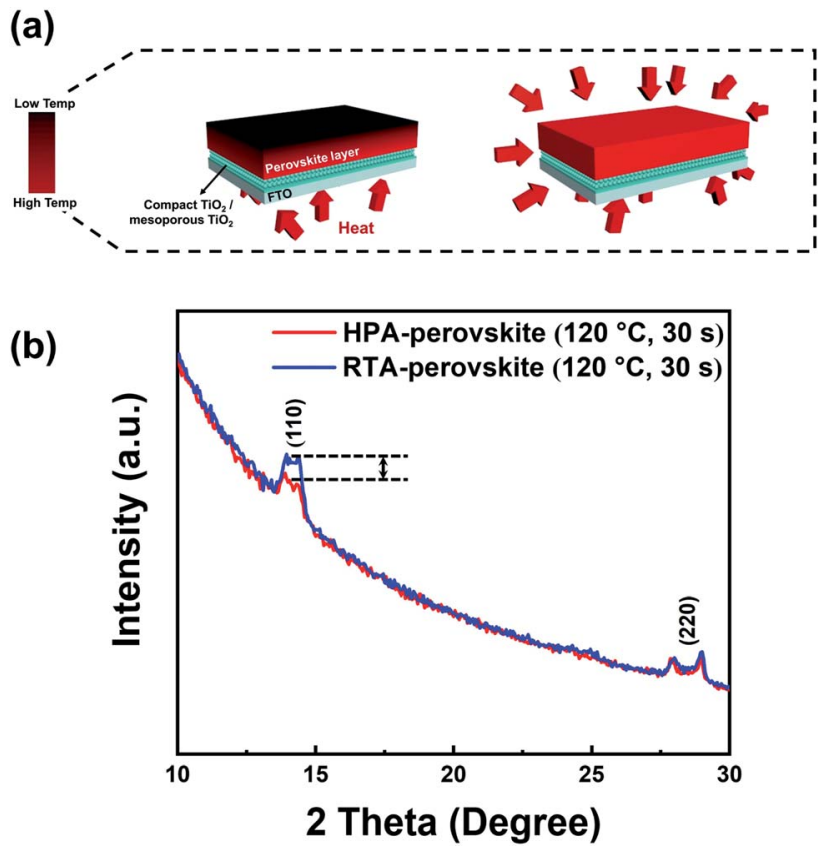

Fig. 2 Scheme of heat transfer mechanism in each annealing method and the analysis of perovskite film at the surface region. (a) Illustration of heat transfer mechanism within the perovskite film via the hot-plate (left side) or RTA (right side) post annealing process. The red arrows indicate the heat transfer direction. (b) GIXRD patterns of two perovskite films prepared by different post-annealing processes. The films were annealed at $120{ }^{\circ} \mathrm{C}$ for only $30 \mathrm{~s}$.

The swift nucleation and crystal growth consequentially contributed to larger grains.

Fig. 3 shows the PV performances of PSCs. Fig. 3a compares representative current density-voltage $(J-V)$ curves, external quantum efficiency (EQE) spectra and the corresponding integrated current density of the PSCs produced by two different post-annealing methods. The best and average PV parameters, including the open-circuit voltage $\left(V_{\mathrm{oc}}\right)$, short-circuit current

(a)
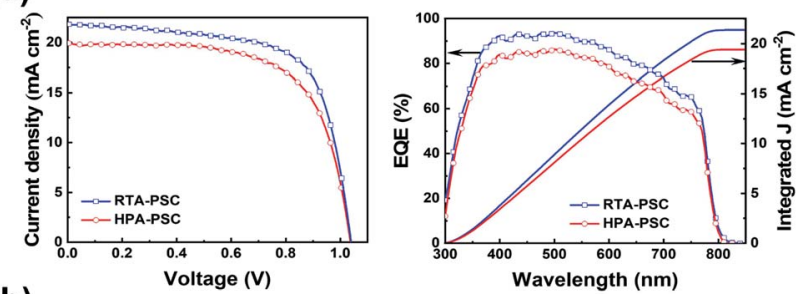

(b)
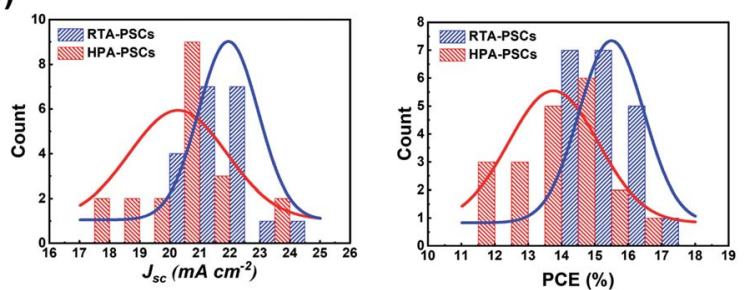

Fig. 3 (a) Representative PV performances of the PSCs prepared by two different post-annealing methods; $J-V$ curves, EQE spectra, and corresponding integrated current density. (b) Histograms of the $J_{\mathrm{sc}}$ and PCE from the 20 PSCs in each annealing method. 
Table 1 Best photovoltaic (PV) parameters including the open-circuit voltage $\left(V_{\text {oc }}\right)$, short-circuit current density $\left(J_{s c}\right)$, fill factor $(F F)$ and PCE. The values in parentheses are the average and standard deviation derived from 20 PSCs

\begin{tabular}{llllrr}
\hline & $V_{\mathrm{oc}}(\mathrm{V})$ & $J_{\mathrm{sc}}\left(\mathrm{mA} \mathrm{cm}^{-2}\right)$ & $\mathrm{FF}$ & $R_{\mathrm{s}}(\Omega)$ & PCE $(\%)$ \\
\hline HPA-PSCs & $1.04(1.04 \pm 0.03)$ & $23.20(20.26 \pm 1.58)$ & $0.67(0.65 \pm 0.03)$ & 106.68 & $16.02(13.74 \pm 1.31)$ \\
RTA-PSCs & $1.06(1.06 \pm 0.02)$ & $24.33(21.94 \pm 0.97)$ & $0.68(0.67 \pm 0.02)$ & 99.48 & $17.37(15.49 \pm 0.95)$ \\
\hline
\end{tabular}

density $\left(J_{\mathrm{sc}}\right)$, fill factor $(\mathrm{FF})$ and PCE, are listed in Table 1 . The PSCs using the HPA-perovskite (HPA-PSCs) demonstrated average PV parameter values, which were obtained from 20 PSCs (the histograms of $J_{\mathrm{sc}}$ and PCE are shown in Fig. $3 \mathrm{~b}$ ); $V_{\mathrm{oc}}$ of $1.04 \mathrm{~V}, J_{\mathrm{sc}}$ of $20.26 \mathrm{~mA} \mathrm{~cm}^{-2}$, FF of 0.65 and PCE of $13.74 \%$. In comparison, the PSCs using RTA-perovskite (RTA-PSCs) demonstrated enhanced PV performances with a $V_{\text {oc }}$ of $1.06 \mathrm{~V}$, a $J_{\text {sc }}$ of $21.94 \mathrm{~mA} \mathrm{~cm}^{-2}$, a FF of 0.67 , a PCE of $15.49 \%$ and improved EQE in visible wavelengths. As explained earlier, the small amount of $\mathrm{PbI}_{2}$ acted as a passivation layer, contributing to the improvement in $J_{\mathrm{sc}}$ in the RTA-PSCs. Compared with the HPA-PSCs, both $J_{\mathrm{sc}}$ and PCE were enhanced by $10 \%$. In addition, the RTA-PSCs demonstrated a lower series resistance of 99.479 $\Omega$ than the HPA-PSCs $(106.679 \Omega)$ measured under illumination conditions, suggesting that the high crystallinity and large perovskite grains allowed the transport of photogenerated carriers with minimal energy loss along the interfaces, which is advantageous for the higher FF.

The interfacial characteristics of the two PSCs prepared by different post-annealing methods were investigated by electrochemical impedance spectroscopy (EIS). EIS was performed at $V_{\text {oc }}$ of each PSC under dark. Fig. $4 \mathrm{a}$ and b show the Nyquist plots (a)

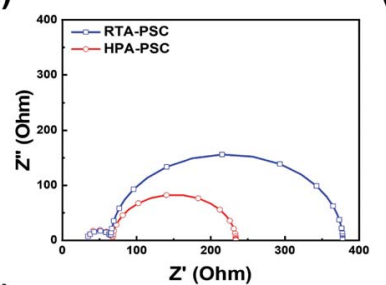

(c)

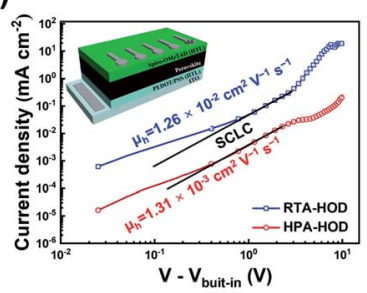

(b)

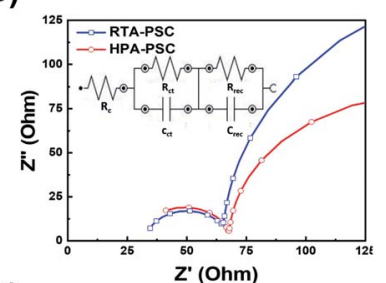

(d)

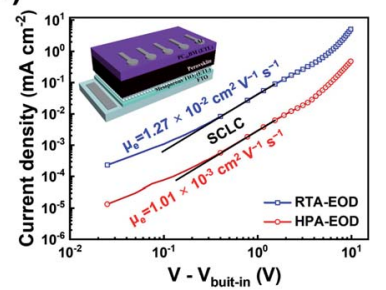

Fig. 4 (a) Nyquist plots of PSCs prepared by two different postannealing methods. The radius of large semi-circle in the lowfrequency region indicates the recombination resistance at the interfaces within the PSC. (b) The magnified Nyquist plots in the highfrequency region; the radius of small semi-circle indicates the charge transport resistance at the interfaces within the PSC. The inset represents the equivalent circuit utilized to fit the Nyquist plots. $J-V$ characteristics of (c) hole-only device and (d) electron-only device. Charge carrier mobility of perovskite film was calculated by fitting the space-charge limited current (SCLC) region with Mott-Gurney law. The device structures of hole-only device and electron-only device are drawn in each graph.

with the equivalent circuit model for interpreting EIS data. The equivalent circuit is shown in the inset of Fig. $4 \mathrm{~b}$. The Nyquist plot is comprised of two semi-circles; the large semi-circle in the low-frequency region below $10 \mathrm{kHz}$ and the small semi-circle in the high-frequency region above $10 \mathrm{kHz}$. The large semi-circle in Fig. 4a is associated with the recombination resistance. Meanwhile, the small semi-circle magnified in Fig. $4 \mathrm{~b}$ relates to the charge-transport resistance. ${ }^{\mathbf{5 0 , 5 1}}$ Herein, $R_{\mathrm{c}}$ represents the contact resistance between the FTO electrode and counter electrode, $R_{\text {rec }}$ and $R_{\text {ct }}$ are defined as the recombination resistance and charge transport resistance at the interfaces, respectively. The values of $R_{\mathrm{c}}, R_{\text {rec }}$ and $R_{\mathrm{ct}}$ are summarized in Table 2 . The $R_{\text {rec }}$ measured in the RTA-PSCs $(312 \Omega)$ was higher than that measured in the HPA-PSCs (166 $\Omega$ ). On the other hand, the RTA-PSCs had a lower $R_{\mathrm{ct}}(32.5 \Omega)$ compared to the HPA-PSCs $(37.6 \Omega)$. The higher $R_{\text {rec }}$ and lower $R_{\text {ct }}$ of the RTAPSCs suggested that charge recombination was greatly suppressed at the interfaces of the HTL and/or electron-transport layer (ETL) with the perovskite film.

Hole and electron mobilities of the two perovskite films were measured with the hole- and electron-only devices; ITO/ PEDOT:PSS/perovskite/spiro-OMeTAD/Ag and fluorine-doped tin oxide (FTO)/TiO 2 blocking layer/mesoporous $\mathrm{TiO}_{2}$ (mp$\mathrm{TiO}_{2}$ )/perovskite/ $\mathrm{PC}_{60} \mathrm{BM} / \mathrm{Al}$, respectively. The hole and electron mobilities can be calculated in the SCLC regime, where the current depends on only the charge carrier mobility and $J-V$ characteristics become quadratic, following the Mott-Gurney law:

$$
J=\frac{9}{8} \mu \varepsilon_{0} \varepsilon_{\text {perov }} \frac{\left(V-V_{\text {built-in }}\right)^{2}}{L^{3}}
$$

where $\mu$ is the carrier mobility, $\varepsilon_{0}$ is the vacuum permittivity, $\varepsilon_{\text {perov }}$ is the relative dielectric constant of perovskite, $L$ is the perovskite film thickness and $V_{\text {built-in }}$ is a correction term to compensate internal built-in fields due to the electrode asymmetry. ${ }^{52-55}$ Fig. $4 \mathrm{c}$ and d present the $J-V$ characteristics of holeand electron-only devices, which are corrected by the $V_{\text {built-in }}$. The mobilities were measured from the slope of fitting line in the SCLC region, following the quadratic dependence on $V$ -

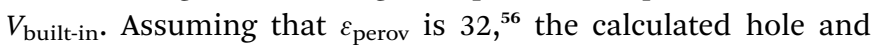

Table 2 Contact resistance $\left(R_{\mathrm{c}}\right)$, recombination resistance $\left(R_{\text {rec }}\right)$ and charge transport resistance $\left(R_{\mathrm{ct}}\right)$ of PSCs obtained by fitting the Nyquist plots to the equivalent circuit

HPA-perovskite RTA-perovskite

Contact resistance, $R_{\mathrm{c}}(\Omega)$

Recombination resistance, $R_{\text {rec }}(\Omega)$

Charge transport resistance, $R_{\mathrm{ct}}(\Omega)$
30.1

166

37.6
33.0

312

32.5 
electron mobilities of RTA-perovskite were $1.26 \times 10^{-2} \mathrm{~cm}^{2} \mathrm{~V}^{-1}$ $\mathrm{s}^{-1}$ and $1.27 \times 10^{-2} \mathrm{~cm}^{2} \mathrm{~V}^{-1} \mathrm{~s}^{-1}$, respectively, which were one order magnitude higher than those $\left(1.31 \times 10^{-3} \mathrm{~cm}^{2} \mathrm{~V}^{-1} \mathrm{~s}^{-1}\right.$ and $1.01 \times 10^{-3} \mathrm{~cm}^{2} \mathrm{~V}^{-1} \mathrm{~s}^{-1}$, respectively) of the HPAperovskite. As carrier mobility is strongly related to crystallinity and grain size of perovskite film, the higher hole and electron mobilities indicate that the RTA-perovskite film has better crystallinity with a lower defect density and less GBs, which limit charge transport by inducing energetic disorder ${ }^{57,58}$ and acting as recombination centers..$^{\mathbf{1 9 , 5 9 , 6 0}}$ These results are well matched with lower series resistance $\left(R_{\mathrm{S}}\right)$ measured in $J-V$ curves (see Table 1).

The photocurrent of PSCs strongly depends on the exciton dissociation efficiency in the perovskite film and this exciton dissociation efficiency is determined by the exciton binding energy. Fig. 5 shows the integrated photoluminescence (PL) intensity of HPA- and RTA-perovskite films at the temperatures from $60 \mathrm{~K}$ to $200 \mathrm{~K}$, which were integrated from the PL spectra in Fig. S3 (ESI $\dagger$ ). The exciton binding energy of perovskite film can be estimated by fitting temperature-dependent integrated PL intensity with following Arrhenius equation:

$$
I(T)=\frac{I_{0}}{1+\mathrm{e}^{-E_{\mathrm{b}} / k_{\mathrm{B}} T}}
$$

where $I_{0}, E_{\mathrm{b}}$ and $k_{\mathrm{B}}$ are the integrated PL intensity at $0 \mathrm{~K}$, the exciton binding energy and the Boltzmann constant, respectively. An exciton binding energies $\left(E_{\mathrm{b}}\right)$ of $65.91 \mathrm{meV}$ and 46.06 meV were calculated for the HPA-perovskite and the RTAperovskite films, respectively and they are within the range of previously reported values for organic-inorganic hybrid perovskite films. ${ }^{61-63}$ The smaller exciton binding energy of the RTAperovskite enabled more electrons and holes to be generated, leading to a higher photocurrent by easy exciton dissociation.

The transient PL characteristics of perovskite films were also measured to evaluate the exciton dissociation efficiency. The PL decay curves (Fig. 6) were monitored at $775 \mathrm{~nm}$, which is the PL emission peak of perovskite film (inset of Fig. 6). The PL decay curves were fitted by bi-exponential function to extract the lifetimes $(\tau)$ and fractional intensities $(f)$ for fast-decay $\left(\tau_{1}, f_{1}\right)$ and slow-decay $\left(\tau_{2}, f_{2}\right)$ components and the intensity-weighted average exciton lifetime ( $\tau_{\text {ave }}$ ) (see Table 3 ). Compared to the $\tau_{\text {ave }}(226 \mathrm{~ns})$ of HPA-perovskite, the $\tau_{\text {ave }}$ of RTA-perovskite was significantly shortened to $136 \mathrm{~ns}$, implying that the generated excitons were efficiently dissociated in the RTA-perovskite, (a)

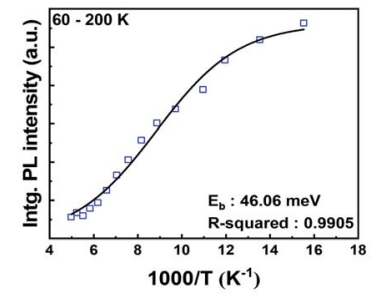

(b)

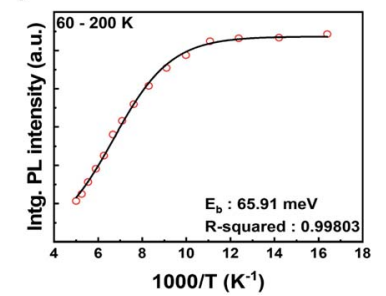

Fig. 5 Temperature dependence of integrated PL intensity of perovskite films; (a) RTA-perovskite and (b) HPA-perovskite. The lines are fitted by Arrhenius equation.

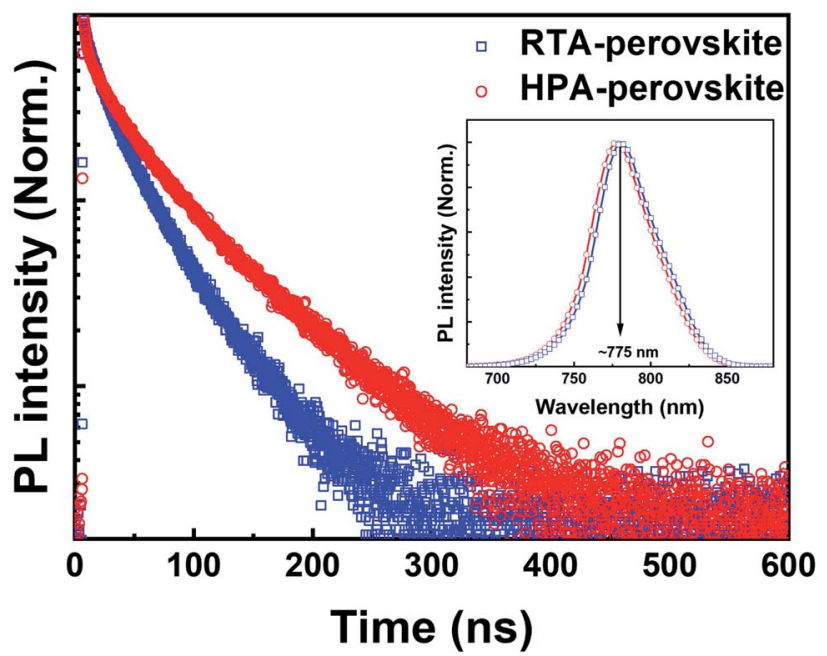

Fig. 6 Time-resolved PL decay curves of the RTA-perovskite and HPA-perovskite prepared on a $\mathrm{mp}-\mathrm{TiO}_{2} /$ glass substrate. The inset shows the steady-state PL spectra.

Table 3 Exciton lifetime of perovskite films annealed by different methods $^{a}$

\begin{tabular}{lllll}
\hline & $\tau_{1}\left(f_{1}\right)(\mathrm{ns})$ & $\tau_{2}\left(f_{2}\right)(\mathrm{ns})$ & $\chi^{2}$ & $\tau_{\text {ave }}(\mathrm{ns})$ \\
\hline HPA-PSCs & $74(0.23)$ & $271(0.77)$ & 1.146 & 226 \\
RTA-PSCs & $55(0.30)$ & $170(0.70)$ & 1.106 & 136
\end{tabular}

${ }^{a}$ The monitored PL emission peak of both perovskite films was $\lambda=$ $775 \mathrm{~nm}$. The PL decay curves were fitted with a bi-exponential function to calculate the lifetime of perovskite. $\chi^{2}$ is the reduced chisquared value. Intensity-weighted average lifetime $\left(\tau_{\text {ave }}\right)$ is defined as $f_{1} \tau_{1}+f_{2} \tau_{2}$, in which $f_{1}$ and $f_{2}$ are fractional intensities and $\tau_{1}$ and $\tau_{2}$ are lifetimes.

enabling more electrons to contribute to external current in PSCs. The excitons with less binding energy can dissociate efficiently, resulting in a short exciton lifetime.

\section{Conclusions}

The perovskite film annealed at $120{ }^{\circ} \mathrm{C}$ for $10 \mathrm{~min}$ by RTA revealed high crystallinity with a larger grain size of $500 \mathrm{~nm}$. The electrochemical impedance spectroscopy (EIS) and space-charge limited current (SCLC) measurements confirmed higher recombination resistance, lower charge transport resistance and higher hole and electron mobilities in RTA-PSCs compared to the HPAPSCs. In addition, a lower exciton binding energy and short exciton lifetime were also achieved from the perovskite film prepared by RTA. Thereby, the PSCs with the RTA-perovskite film demonstrated enhanced $J_{\mathrm{sc}}$ and PCE by $10 \%$, compared to the HPA-perovskite PSCs. This study provides a simple method for achieving perovskite films with high crystallinity and large grain size for realizing highly efficient PSCs.

\section{Conflicts of interest}

There are no conflicts to declare. 


\section{Acknowledgements}

This work was supported by the Pioneer Research Center Program (NRF-2016M3C1A3908893, NRF-2014M3C1A3016468) and by the Basic Science Research Program (NRF2016R1A2B4006395) through the National Research Foundation of Korea (NRF) funded by the Ministry of Education. G. Y. Jung was partially supported by the GIST Research Institute (GRI) project through a grant provided by GIST in 2018. C.-L. Lee thanks the National Research Foundation of Korea (NRF) for financial support through a grant funded by the Korean government (MSIP; NRF-2016R1A2B4013003 and NRF2018R1A2A3075144) and a grant funded by GIST 2019 (Research on Advanced Optical Science and Technology).

\section{Notes and references}

1 N. J. Jeon, J. H. Noh, W. S. Yang, Y. C. Kim, S. Ryu, J. Seo and S. I. Seok, Nature, 2015, 517, 476.

2 J.-H. Im, C. R. Lee, J.-W. Lee, S. W. Park and N. G. Park, Nanoscale, 2011, 3, 4088.

3 N. G. Park, J. Phys. Chem. Lett., 2015, 4, 2423.

4 N. G. Park, Nano Convergence, 2016, 3, 15.

5 E. Mosconi, A. Amat, M. K. Nazeeruddin, M. Gratzel and F. D. Angelis, J. Phys. Chem. C, 2013, 117, 13902.

6 G. Xing, N. Mathews, S. Sun, S. S. Lim, Y. M. Lam, M. Gratzel, S. Mhaisalkar and T. C. Sum, Science, 2013, 342, 344.

7 H. Kim, K.-G. Lim and T.-W. Lee, Energy Environ. Sci., 2016, 9, 12.

8 J. H. Heo, D. H. Song, H. J. Han, S. Y. Kim, J. H. Kim, D. Kim, H. W. Shin, T. K. Ahn, C. Wolf, T.-W. Lee and S. H. Im, Adv. Mater., 2015, 27, 3424.

9 B. Jeong, S. M. Cho, S. H. Cho, J. H. Lee, I. Hwang, S. K. Hwang, J. Cho, T.-W. Lee and C. Park, Phys. Status Solidi RRL, 2016, 10, 381.

10 A. Kojima, K. Teshima, Y. Shirai and T. Miyasaka, J. Am. Chem. Soc., 2009, 131, 6050.

11 H.-S. Kim, C.-R. Lee, J.-H. Im, K.-B. Lee, T. Moehl, A. Marchioro, S.-J. Moon, R. Humphry-Baker, J.-H. Yum, J. E. Moser, M. Gratzel and N.-G. Park, Sci. Rep., 2012, 2, 591.

12 M. M. Lee, J. Teuscher, T. Miyasaka, T. N. Murakami and H. J. Snaith, Science, 2012, 338, 643.

13 M. Liu, M. B. Johnston and H. J. Snaith, Nature, 2013, 501, 395.

14 T. Leijtens, B. Lauber, G. E. Eperon, S. D. Stranks and H. J. Snaith, J. Phys. Chem. Lett., 2014, 5, 1096.

15 Z. Xiao, C. Bi, Y. Shao, Q. Dong, Q. Wang, Y. Yuan, C. Wang, Y. Gao and J. Huang, Energy Environ. Sci., 2014, 7, 2619.

16 W. S. Yang, J. H. Noh, N. J. Jeon, Y. C. Kim, S. Ryu, J. Seo and S. I. Seok, Science, 2015, 348, 1234.

17 W. S. Yang, B.-W. Park, E. H. Jung, N. J. Jeon, Y. C. Kim, D. U. Lee, S. S. Shin, J. Seo, E. K. Kim, J. H. Noh and S. I. Seok, Science, 2017, 356, 1376.

18 J. H. Noh, S. H. Im, J. H. Heo, T. N. Mandal and S. I. Seok, Nano Lett., 2013, 13, 1764.

19 W. Nie, H. Tsai, R. Asadpour, J.-C. Blancon, A. J. Neukirch, G. Gupta, J. J. Crochet, M. Chhowalla, S. Tretiak,
M. A. Alam, H.-L. Wang and A. D. Mohite, Science, 2015, 347, 522.

20 F. Giordano, A. Abate, J. P. C. Baena, M. Saliba, T. Matsui, S. H. Im, S. M. Zakeeruddin, M. K. Nazeeruddin, A. Hagfeldt and M. Gratzel, Nat. Commun., 2016, 7, 10379.

21 A. Miyate, A. Mitioglu, P. Plochocka, O. Portugall, J. T.-W. Wang, S. D. Strank, H. J. Snaith and R. J. Nicholas, Nat. Phys., 2015, 11, 582.

22 G. Horowitz and M. E. Hajlaoui, Adv. Mater., 2000, 12, 1046. 23 Y. Zhao, A. M. Nardes and K. Zhu, J. Phys. Chem. Lett., 2014, 5, 490 .

24 L. Zheng, Y. Ma, S. Chu, S. Wang, B. Qu, L. Xiao, Z. Chen, Q. Gong, Z. Wu and X. Hou, Nanoscale, 2014, 6, 8171.

25 J.-H. Im, I.-H. Jang, N. Pellet, M. Gratzel and N.-G. Park, Nat. Nanotechnol., 2014, 9, 927.

26 Z. Xiao, Q. Dong, C. Bi, Y. Shao, Y. Yuan and J. Huang, Adv. Mater., 2014, 26, 6503.

27 J.-J. Li, J.-Y. Ma, Q.-Q. Ge, J.-S. Hu, D. Wang and L.-J. Wan, ACS Appl. Mater. Interfaces, 2015, 7, 28518.

28 N. J. Jeon, J. H. Noh, Y. C. Kim, W. S. Yang, S. Ryu and S. I. Seok, Nat. Mater., 2014, 13, 897.

29 N. Ahn, D.-Y. Son, I.-H. Jang, S. M. Kang, M. Choi and N.-G. Park, J. Am. Chem. Soc., 2015, 137, 8696.

30 O. Malinkiewicz, A. Yella, Y. H. Lee, G. M. Espallargas, M. Gratzel, M. K. Nazeeruddin and H. J. Bolink, Nat. Photonics, 2014, 8, 128.

31 J. H. Kim, S. T. Williams, N. Cho, C.-C. Chueh and A. K.-Y. Jen, Adv. Energy Mater., 2015, 5, 1401229.

32 T. Jeon, H. M. Jin, S. H. Lee, J. M. Lee, H. I. Park, M. K. Kim, K. J. Lee, B. Shin and S. O. Kim, ACS Nano, 2016, 10, 7907.

33 F. Huang, S. Jin, Y. Wei, L. Gu, Q. Guo, H. Xu, X. Yang, T. Wu, X. He, Y. Huang, L. Fan and J. Wu, Electrochim. Acta, 2018, 259, 402.

34 T. Y. Ma and D. K. Shim, Thin Solid Films, 2002, 410, 8.

35 C.-F. Zhu, W.-K. Fong, B.-H. Leung, C.-C. Cheng and

C. Surya, IEEE Trans. Electron Devices, 2001, 48, 1225.

36 B. W. Lavery, S. Kumari, H. Konermann, G. L. Draper, J. Spurgeon and T. Druffel, ACS Appl. Mater. Interfaces, 2016, 8, 8419.

37 V. L. Pool, B. Dou, D. G. Van Campen, T. R. K-Stockert, F. S. Barnes, S. E. Shaheen, Md. I. Ahmad, M. F. A. M. van Hest and M. F. Toney, Nat. Commun., 2017, 8, 14075.

38 B. Dou, V. L. Pool, M. F. Toney and M. F. A. M. van Hest, Chem. Mater., 2017, 29, 5931.

39 J. Feng, X. Zhu, Z. Yang, X. Zhang, J. Niu, J. Niu, Z. Wang, S. Zuo, S. Priya, S. Liu and D. Yang, Adv. Mater., 2018, 30, 1801418.

40 N. Pellet, P. Gao, G. Gregori, T.-Y. Yang, M. K. Nazeeruddin, J. Maier and M. Gratzel, Angew. Chem., Int. Ed., 2014, 53, 3151.

41 G. E. Eperon, C. E. Beck and H. J. Snaith, Mater. Horiz., 2016, 3, 63.

42 T. Leijtens, G. E. Eperon, A. J. Barker, G. Grancini, W. Zhang, J. M. Ball, A. R. S. Kandada, H. J. Snaith and A. Petrozza, Energy Environ. Sci., 2016, 9, 3472.

43 C. Zhang, Q. Luo, J. Shi, L. Yue, Z. Wang, X. Chen and S. Huang, Nanoscale, 2017, 9, 2852. 
44 M. Jiang, J. Wu, F. Lan, Q. Tao, D. Gao and G. Li, J. Mater. Chem. A, 2015, 3, 963.

45 Q. Chen, H. Zhou, T.-B. Song, S. Luo, Z. Hong, H.-S. Duan, L. Dou, Y. Liu and Y. Yang, Nano Lett., 2014, 14, 4158.

46 L. Wang, C. McCleese, A. Kovalsky, Y. Zhao and C. Burda, J. Am. Chem. Soc., 2014, 136, 12205.

47 S. Song, M. T. Horantner, K. Choi, H. J. Snaith and T. Park, J. Mater. Chem. A, 2017, 5, 3812.

48 Z. Liang, S. Zhang, X. Xu, N. Wang, J. Wang, X. Wang, Z. Bi, G. Xu, N. Yuan and J. Ding, RSC Adv., 2015, 5, 60562.

49 P. Colombi, P. Zanola, E. Bontempi, R. Roberti, M. Gelfi and L. E. Depero, J. Appl. Crystallogr., 2006, 39, 176.

50 Z. Zhu, X. Zheng, Y. Bai, T. Zhang, Z. Wang, S. Xiao and S. Yang, Phys. Chem. Chem. Phys., 2015, 17, 18265.

51 G. Niu, W. Li, F. Meng, L. Wang, H. Dong and Y. Qiu, J. Mater. Chem. A, 2014, 2, 705.

52 A. Kahn, N. Koch and W. Gao, J. Polym. Sci., Part B: Polym. Phys., 2003, 41, 2529.

53 J. Hwang, A. Wan and A. Kahn, Mater. Sci. Eng., R, 2009, 64, 1. 54 M. Kiy, P. Losio, I. Biaggio, M. Koehler, A. Tapponnier and P. Gunter, Appl. Phys. Lett., 2002, 80, 1198.
55 W. Chandra, L. K. Ang, K. L. Pey and C. M. Ng, Appl. Phys. Lett., 2007, 90, 153505.

56 Q. Dong, Y. Fang, Y. Shao, P. Mulligan, J. Qiu, L. Cao and J. Huang, Science, 2015, 347, 967.

57 J. You, Y. Yang, Z. Hong, T.-B. Song, L. Meng, Y. Liu, C. Jiang, H. Zhou, W.-H. Chang, G. Li and Y. Yang, Appl. Phys. Lett., 2014, 105, 183902.

58 G. E. Eperon, V. M. Burlakov, P. Docampo, A. Goriely and H. J. Snaith, Adv. Funct. Mater., 2014, 24, 151.

59 D. Li, S. A. Bretschneider, V. W. Bergmann, I. M. Hermes, J. Mars, A. Klasen, H. Lu, W. Tremel, M. Mezger, H.-J. Butt, S. A. L. Weber and R. Berger, J. Phys. Chem. C, 2016, 120, 6363.

60 Z. Xiao, Q. Dong, C. Bi, Y. Shao, Y. Yuan and J. Huang, Adv. Mater., 2014, 26, 6503.

61 N. Sestu, M. Cadelano, V. Sarritzu, F. Chen, D. Marongiu, R. Piras, M. Mainas, F. Quochi, M. Saba, A. Mura and G. Bongiovanni, J. Phys. Chem. Lett., 2015, 6, 4566.

62 K. Wu, A. Bera, C. Ma, Y. Du, Y. Yang, L. Li and T. Wu, Phys. Chem. Chem. Phys., 2014, 16, 22476.

63 J. Dai, H. Zheng, C. Zhu, J. Lu and C. Xu, J. Mater. Chem. C, 2016, 4, 4408. 